\title{
Introducing stress random fields of polycrystalline aggregates into the local approach to fracture
}

\section{Conference Paper}

Author(s):

Dang, X.H.; Berveiller, Marc; Zeghadi, Asmahana; Sudret, Bruno (D); Yalamas, Thierry

Publication date:

2013

Permanent link:

https://doi.org/10.3929/ethz-a-010057944

Rights / license:

In Copyright - Non-Commercial Use Permitted 


\title{
Introducing stress random fields of polycrystalline aggregates into the local approach to fracture
}

\author{
X.H. Dang \\ Faculty of Building and Industrial Construction \\ National University of Civil Engineering, Hanoi, Vietnam. \\ M. Berveiller \& A. Zeghadi \\ Departement of Material and Mechanics of Components \\ EDF R\&D, Moret Sur Loing, France. \\ B. Sudret \\ ETH Zürich, Institute of Structural Engineering, Chair of Risk, Safety \& Uncertainty Quantification \\ Wolfgang-Pauli-Strasse 15, CH-8093 Zürich \\ T. Yalamas \\ Phimeca Engineering SA, Paris, France.
}

\begin{abstract}
The coupling of the local approach to fracture and the polycrystalline aggregates simulations is investigated in this paper. A series of Representative Volume Elements (RVEs) drawing randomly the crystallographic orientations and the grain geometries are firstly simulated. Then the resulting realizations of the principal stress random field are used in order to identify the properties of a Gaussian random field using the periodogram approach. The anisotropy of the stress random field is observed and the correlation lengths are identified. Then the synthetic realizations of the identified principal stress random field are simulated using the spectral simulation method. The weakest link theory is lastly applied with this identified principal stress field and simulations of carbides distributions.
\end{abstract}

\section{INTRODUCTION}

The brittle fracture behavior of the A508 steel has been thoroughly studied for a long time using the multiscale analysis of material properties. At the mesoscopic scale, one realized a serie of failure tests on specimens and estimated an experimental failure probability curve. In another way, a mechanical modeling of the specimen was realized to apply the local approach to fracture theory [1] and the so-called Beremin model [2], which assumes that cleavage is controlled by the propagation of the weakest link between a population of pre-existing micro-defects in the material. The fitting of the failure probability curve by the Beremin model gave us the brittle properties of the material.

This approach has been recently coupled with polycrystalline aggregates simulations at the microscopic scale [3]. The main idea of such an approach is to model a single material representative volume element (RVE) as a polycrystalline aggregate and compute the principal stress field under given load condi- tions. Then a statistical distribution of defects (carbides) is sampled over the volume. In each Gauss point of the finite element mesh the cleavage criterion is attained somewhere along the load path if a Griffith-like criterion applied to the size of the carbide in this Gauss point is reached (cleavage propagation). Within the weakest link theory the failure of a single critical carbide induces the failure of the RVE. Then the failure probability curve of the RVE is fitted using the Beremin model $[3,4]$ to explain the dependence on temperature of material properties. However it is believed that numerous parameters such as grain geometry and orientation may influence the stress field and thus the final result.

In this paper we propose to simulate a series of RVEs by drawing randomly the crystallographic orientations and the grain geometries of the material. The resulting realizations of the principal stress field are used in order to identify the properties of a Gaussian random field using the periodogram approach $[5,6]$. The anisotropy of the stress random field is observed and correlation lengths are identified. The weakest 
link theory the failure is then applied with this identified cleavage stress field and simulations of carbides distributions.

\section{LOCAL APPROACH TO FRACTURE AT MICROSCOPIC SCALE}

\subsection{Polycrystalline aggregates simulation}

The A508 ferritic steel has a granular microstructure with some ferrite lath packets of different lattice orientations in each grain. The synthetic representation of this microstructure is showed in [7]. In this work, the simplified microstructure is used. The construction of gralunar structure is based on the Voronoi polyhedra model [8], generated in this work with the Quickhull algorithm [9]. The same crystallographic orientation, defined by the three Euler angles $\varphi_{1}, \phi$, $\varphi_{2}$, is randomly assigned to all integration points inside each individual grain using a uniform distribution. The random granular structure and the random crystallographic orientation of each grain are the origin of the randomness of the stress field in the material. Two cases of random microstructure are considered.

- Case 1 named random geometry (RG): Both the granular structure and the crystallographic orientations are randomly simulated.

- Case 2 named fixed geometry (FG): The granular structure is fixed, only the crystallographic orientations are randomly simulated.

Figure 1 shows a polycrystalline aggregate covering a square volume of size 1000 containing 100 grains. The random crystallographic orientations are represented by different colors in each grain. Ferrite has a body centered cubic (BCC) structure. Three families of slip systems should be taken into account, namely $\{110\}\langle 111\rangle,\{112\}\langle 111\rangle,\{123\}\langle 111\rangle$. However, following [10] it is assumed that the glides on the plane 123 are a succession of micro-glides on the planes 110,112 . This leads to consider only the two first families, which yields 24 slip systems by symmetry. The model for crystal plasticity chosen in this work has been originally formulated in [11] within the small strain framework. The total strain rate $\dot{\varepsilon}_{i j}$ is classically decomposed as the sum of the elastic strain rate $\dot{\varepsilon}_{i j}^{e}$ and plastic strain rate $\dot{\varepsilon}_{i j}^{p}$.

$\dot{\varepsilon}_{i j}=\dot{\varepsilon}_{i j}^{e}+\dot{\varepsilon}_{i j}^{p}$

The elastic part follows the Hooke's law and the plastic part is calculated from the shear strain rates of the 24 active slip systems.

$\dot{\varepsilon}_{i j}^{p}=\sum_{g=1}^{24} \dot{\gamma}^{g} R_{i j}^{g}$ where $\dot{\gamma}^{g}$ is the shear strain rate of the slip system $g$ and $R_{i j}^{g}$ is the Schmid factor which is the geometrical projection tensor. The latter is calculated from the normal vector to the gliding plane $\mathbf{n}$ and the direction of gliding $\mathbf{m}$.

$R_{i j}^{g}=\frac{1}{2}\left(m_{i} n_{j}+m_{j} n_{i}\right)$

The Resolved Shear Stress (RSS) $\tau^{g}$ of the slip system $g$ is the projection of the stress tensor via the Schmid factor.

$\tau^{g}=R_{i j}^{g} \sigma_{i j}$

The shear strain rates $\dot{\gamma}^{g}$ of each slip system $g$ are the internal variable that describes plasticity. The evolution of these variables depends on the difference between the RSS $\tau^{g}$ and the actual critical RSS $\tau_{c}^{g}$ in an elastoviscoplastic setting:

$\dot{\gamma}^{g}=\left(\frac{\tau^{g}-\tau_{c}^{g}}{K}\right)^{n} \operatorname{sign}\left(\tau^{g}\right)$

where $K$ and $n$ are material constants, and $\operatorname{sign}(a)=$ $a /|a|$ if $a \neq 0$ and 0 otherwise. The critical $\operatorname{RSS} \tau_{c}^{g}$ evolves according to the following isotropic hardening law:

$\tau_{c}^{g}=\tau_{c 0}^{g}+Q^{g} \sum_{s=1}^{24} h^{g s}\left(1-e^{-b^{g} \gamma_{c u m}^{s}}\right)$

where $\gamma_{\text {cum }}^{s}=\int_{t_{0}}^{t}\left|\dot{\gamma}^{s}\right| d t$. The exponential term presents the hardening saturation in the material when the accumulated slip is high. $\tau_{c 0}^{g}$ is the initial critical RSS on the considered system $g . Q^{g}$ and $b^{g}$ are parameters which depend on the material. $h^{g s}$ is the hardening matrix of size $24 \times 24$ whose component $h^{g s}$ presents the hardening effect of the system $g$ on the system $s$. The values of these coefficients and this matrix are presented in [6].

A tensile test on a bidimensional polycrystalline aggregate is simulated under plane strain conditions and the grain boundaries considered as perfect interfaces. The boundary conditions applied onto the aggregate are sketched in Figure 1. The lower surface is blocked along the $Y$ direction. The displacements $D X=D Y=0$ are blocked at the origin of the coordinate system (lower left corner). On the upper surface, a homogeneous displacement is applied by steps in the $Y$ direction up to a macroscopic strain equal to $3.5 \%$. The computation is carried out using the open source finite element software Code_Aster (http://www.code-aster.org).

\subsection{Local approach to fracture}

The result of the polycrystalline aggregate simulation is the principal stress field $\sigma_{I}$ in the Gauss points at 


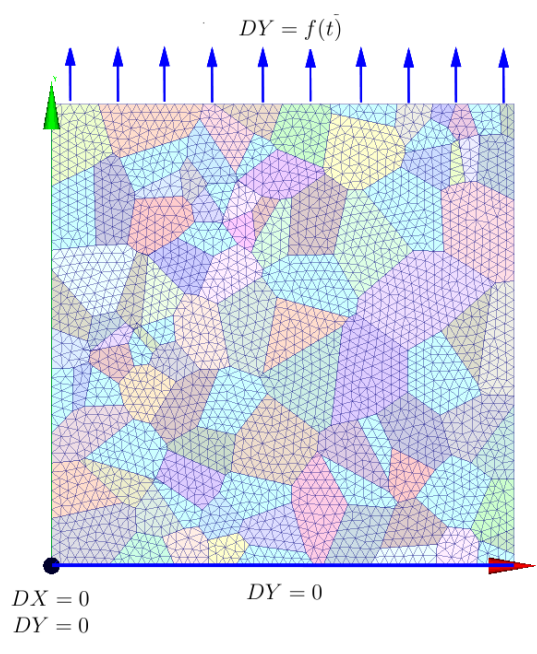

Figure 1: Two-dimensional polycrystalline aggregate modeling a volume of A508 steel (100 grains, size 1000 x 1000) and the boundary conditions.

different increase load levels $\left\{\varepsilon_{1}, \ldots, \varepsilon_{n}\right\}$. In this approach, the specimen is considered as representative. Thus a single simulation is realized.

At the Gauss points, $N$ statistical distributions of defects (carbides size) $r_{c}$ are sampled over the volume. The cleavage is assumed to be controlled by the propagation of the weakest link between the sampled micro-defects at the Gauss points. The $N$ samples of resistance stress field $\sigma_{c}$ is calculated by the Griffith criterion [12].

$\sigma_{c}=\sqrt{\frac{\pi E \gamma_{p}}{2\left(1-\nu^{2}\right) r_{c}}}$

where

$\gamma_{p}$ is the effective surface energy.

$E, \nu$ are the material's coefficients.

Now for each sample $\sigma_{c}^{i}$, this is compared with the principal stress field at each load level $\sigma_{I}^{j}$ in increasing order. By interpolation, one can compute the load level $\varepsilon_{i}^{r}$ that corresponds to failure, i.e. where $\sigma_{c}^{i}=\sigma_{I}$. The process if repeated for all $N$ samples of the resistance stress field and $N$ failure load levels $\left\{\varepsilon_{1}^{r}, \ldots, \varepsilon_{N}^{r}\right\}$ are noted. The empirical distribution function of this failure load level set shows the failure probability of the specimen in increasing load level. The detail of this approach is showed in [7, 3]. Figure 2 shows the 20 failure probability curves estimated from 20 bi-dimensional specimen simulations with 20 different microstructures using this approach.

It is showed that the variability of the microstructure that is represented by the stress random field, influence the failure probability estimation. Thus this variability is needed to be taken into account in the local approach to fracture to estimate the failure probability of the specimen accurately.

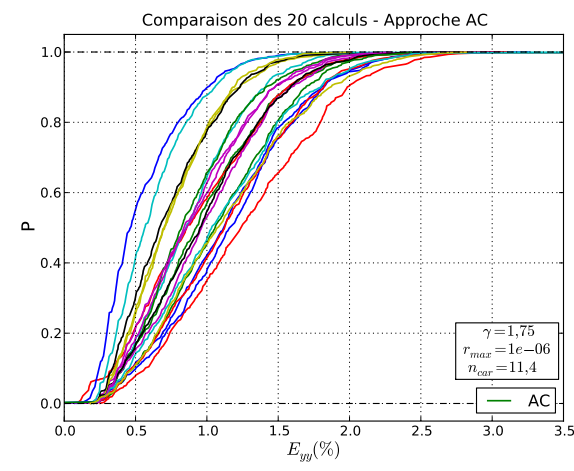

Figure 2: Failure probability curves estimated from 20 polycrystalline aggregate simulations with 20 different microstructures.

\section{PRINCIPAL STRESS RANDOM FIELD}

\subsection{Randomness of principal stress field}

The variability of the granular structure and the crystallographic orientations leads the randomness of the principal stress field in the polycrystalline aggregate. Thus each polycrystalline aggregate simulation of a given microstructure gives a realization of the principal stress random field. In this work, this stress random field is assumed to be an ergodic stationary Gaussian random field. The identification of such a random field is to identify its constant mean $\mu$, standard deviation $\sqrt{v}$ and its correlation spatial structure $C\left(\boldsymbol{h}=\left|\boldsymbol{x}-\boldsymbol{x}^{\prime}\right|\right)$. The mean value is directly estimated as the numerical mean of the realizations of the field. The others are estimated using the periodogram method.

In this work, 40 polycrystalline aggregate simulations are carried out for each case of the simulated microstructure, namely RG and FG. In order to apply the periodogram method and to avoid the boundary effect, the principal stress random field has to be projected on a regular grid of $256 \times 256$ points in the domain $800 \times 800$ in the center of the specimen. In this paper only the case RG is showed. The other case is detailed in [6].

\subsection{Periodogram approach}

The Power Spectral Density (PSD) function of a stationary random field is defined as the Fourier transformation of its covariance function. Its estimation from a realization of the random field is called the periodogram. In the bi-dimensional case, the average periodogram estimated from $K$ realizations $\mathcal{X}_{i}=$ $\left\{h^{i}\left(x_{m}, y_{n}\right), m=0, \ldots, M-1, n=0, \ldots, N-1\right\}$, $i=1, \ldots, K$ assumed independent of the random field reads:

$$
\begin{aligned}
& \hat{P}\left(f_{x j}, f_{y k}\right)=\frac{1}{K M N U} \times \\
& \left|\sum_{m=0}^{M-1} \sum_{n=0}^{N-1}\left[w(m, n) h^{i}\left(x_{m}, y_{n}\right)\right] e^{-i 2 \pi\left(\frac{m j}{2 M}+\frac{n k}{2 N}\right)}\right|^{2}
\end{aligned}
$$


where $w(m, n)$ is the filter window $[13,14]$ and $U$ is the spectral power of the window that is calculated by:

$U=\frac{1}{M N} \sum_{m=0}^{M-1} \sum_{n=0}^{N-1}|w(m, n)|^{2}$

In practice, the point number of the grid $(M, N)$ are usually chosen as powers of two. The detail of this method is showed in $[5,6,14,15]$.

\subsection{Identification of a Gaussian random field}

The full identification process is presented below. The detail of each step can be found in [6].

- Step 1: Verify the assumption of normality of the principal stress random field.

- Step 2: Verify the stationarity of the random field.

- Step 3: Identification of the random field properties at a given load level.

- Step 4: Identification of the random field properties at all the load levels.

The Gaussian and stationarity assumption (steps 1,2) of the random field proved acceptable. In this paper, one presents only the result of Steps 3 and 4 . One firstly estimate the average periodogram from 40 realizations of the stress field. This one is then fitted with theoretical PSD models by the least square algorithm. Figure 3 presents the estimated average periodogram.

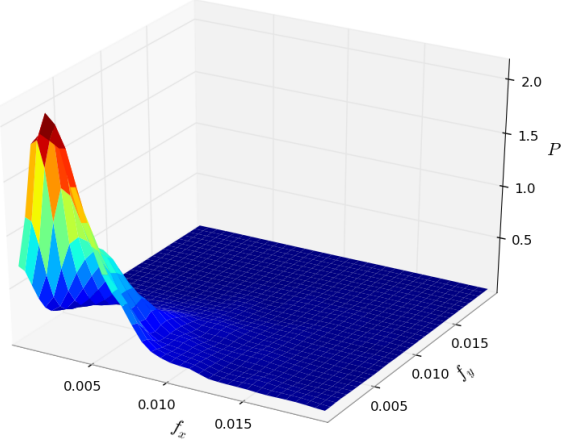

Figure 3: Average empirical periodogram estimated from 40 realizations of the principal stress random field $\sigma_{I}$.

From a visual inspection of the obtained empirical periodograms it appears that a Gaussian $\left(P_{g}\right)$ or an exponential $\left(P_{e}\right)$ model of periodogram such as those presented below may be consistent with the data. However it appeared in the various analyses that the peak of the periodogram is not always at the origin. An initial frequency is thus introduced which shifts the theoretical periodogram. Finally, due to lack of fitting of the single-type periodogram (e.g. Gaussian and exponential), a combination thereof (so-called mixed model $\left.P_{m}\right)$ is also fitted. The most general model finally reads:

$$
\begin{gathered}
P_{g}\left(f_{x}, f_{y}\right)=v_{1} \pi l_{x 1} \exp \left[\pi^{2} l_{x 1}^{2}\left(f_{x}-f_{x 0}^{(1)}\right)^{2}\right] \\
\quad \times l_{y 1} \exp \left[\pi^{2} l_{y 1}^{2}\left(f_{y}-f_{y 0}^{(1)}\right)^{2}\right] \\
P_{e}\left(f_{x}, f_{y}\right)=v_{2} \frac{2 l_{x 2}}{1+4 \pi^{2} l_{x 2}^{2}\left(f_{x}-f_{x 0}^{(2)}\right)^{2}} \\
\quad \times \frac{2 l_{y 2}}{1+4 \pi^{2} l_{y 2}^{2}\left(f_{y}-f_{y 0}^{(2)}\right)^{2}} \\
P_{m}=P_{g}+P_{e}
\end{gathered}
$$

where $v_{1}, v_{2}$ and $l_{x 1}, l_{y 1}, l_{x 2}, l_{y 2}$ are respectively variances and correlation lengths in each direction $X$ and $Y$ (anisotropic field) for each component (1) (Gaussian part) and (2) (exponential part). Similarly $f_{x 0}^{(1)}, f_{y 0}^{(1)}, f_{x 0}^{(2)}, f_{y 0}^{(2)}$ are initial shift frequencies.

Note that Eq. $(10,11,12)$ correspond only to positive values of $f_{x}, f_{y}$. The periodogram is then extended by symmetry for negative frequencies. In terms of associated covariance models, the linear combination of periodograms leads to a linear combination of covariance models. The initial frequency shift in the periodogram leads to oscillatory cosine terms in the covariance by inverse Fourier transform:

$$
\begin{aligned}
& C_{g}=v_{1} \exp \left[-\left(\frac{h_{x}^{2}}{l_{x 1}^{2}}+\frac{h_{y}^{2}}{l_{y 1}^{2}}\right)\right] \cos \left(2 \pi f_{x 0}^{(1)} h_{x}\right) \\
& \times \cos \left(2 \pi f_{y 0}^{(1)} h_{y}\right) \\
& C_{e}=v_{2} \exp \left[-\left(\frac{\left|h_{x}\right|}{l_{x 2}}+\frac{\left|h_{y}\right|}{l_{y 2}}\right)\right] \cos \left(2 \pi f_{x 0}^{(2)} h_{x}\right) \\
& \times \cos \left(2 \pi f_{y 0}^{(2)} h_{y}\right) \\
& C_{m}=C_{g}+C_{e}
\end{aligned}
$$

In order to compare the various fittings the least-square residual between the empirical periodogram $\bar{P}\left(f_{x}, f_{y}\right)$ (Eq.(8)) and the fitted periodogram $P\left(f_{x}, f_{y}\right)$ is finally computed. The following non dimensional error estimate is used:

$$
\bar{\Delta}=\sqrt{\frac{1}{M N} \sum_{i=1}^{N} \sum_{j=1}^{M}\left[\frac{\hat{P}\left(f_{x i}, f_{y j}\right)-P\left(f_{x i}, f_{y j}\right)}{\max (\hat{P})}\right]^{2}}
$$

Table 1 presents the results of the fitting of the average empirical periodogram calculated from 40 realizations of the field using three models, namely 


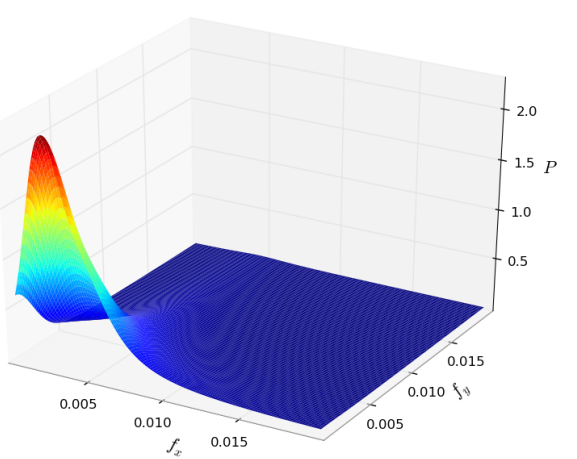

Figure 4: Best fitted periodogram of the principal stress field $\sigma_{I}$ at $3.5 \%$ macroscopic strain (model Gaussian + exponential)

\begin{tabular}{|c|c|c|c|c|}
\hline & Model & Gaussian & exponential & mixed \\
\hline$\Delta$ & 0,004 & 0,004 & 0,002 & \\
\hline \multirow{5}{*}{ 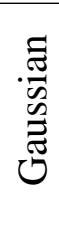 } & $\sqrt{v_{1}}$ & 78,29 & $x$ & 49,10 \\
\hline & $l_{x 1}$ & 99,28 & $x$ & 186,2 \\
\hline & $l_{y 1}$ & 98,46 & $x$ & 127,6 \\
\hline & $f_{x 0}^{(1)}$ & 0,003 & $x$ & 0,002 \\
\hline & $f_{y 0}^{(1)}$ & 0,000 & $x$ & 0,000 \\
\hline \multirow{5}{*}{ 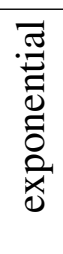 } & $\sqrt{v_{2}}$ & $x$ & 103,4 & 83,87 \\
\hline & $l_{x 2}$ & $x$ & 68,89 & 69,87 \\
\hline & $l_{y 2}$ & $x$ & 74,63 & 50,75 \\
\hline & $f_{x 0}^{(2)}$ & $x$ & 0,003 & 0,005 \\
\hline & $f_{y 0}^{(2)}$ & $x$ & 0,000 & 0,000 \\
\hline
\end{tabular}

Table 1: Fitted parameters and error estimates for the three fitted models: Gaussian, exponential and mixed "Gaussian + exponential"

Gaussian, exponential and a mixed "Gaussian + exponential" as in Eq. $(10,11,12)$. The best fitted periodogram of the maximal principal stress field $\sigma_{I}$ at $3.5 \%$ of macroscopic strain is plotted in Figure 4.

From the results in Table 1 it appears that the mixed model provides a significantly smaller least-square error than that obtained from the Gaussian and exponential models respectively. The corresponding fitted periodogram is plotted in Figure 4.

In order to better appreciate the quality of the fitting, two-dimensional cuts of the empirical (resp. fitted) periodogram are given in Figures 5.

In order to comment the result in the Table 1, let us to define the fluctuation scale $\vartheta=\sqrt{\pi} l$ for the Gaussian component, $\vartheta=2 l$ for the exponential component and the mean size of grains as below:

$D_{g}=\sqrt{\frac{4}{\pi} S_{g}}=\sqrt{\frac{4}{\pi} \frac{1000 \times 1000}{100}}=112.8$

where $S_{g}$ is the mean area of the grains.

The mixed model leads us to think about two parts of the stress field: one in the grains and the other on the grains boundaries. The covariance structure of these two parts are respectively represented by the Gaussian and the exponential components of the identified covariance model. One observes that the exponential component is more dispersed than the other $\left(v_{2}>v_{1}\right)$
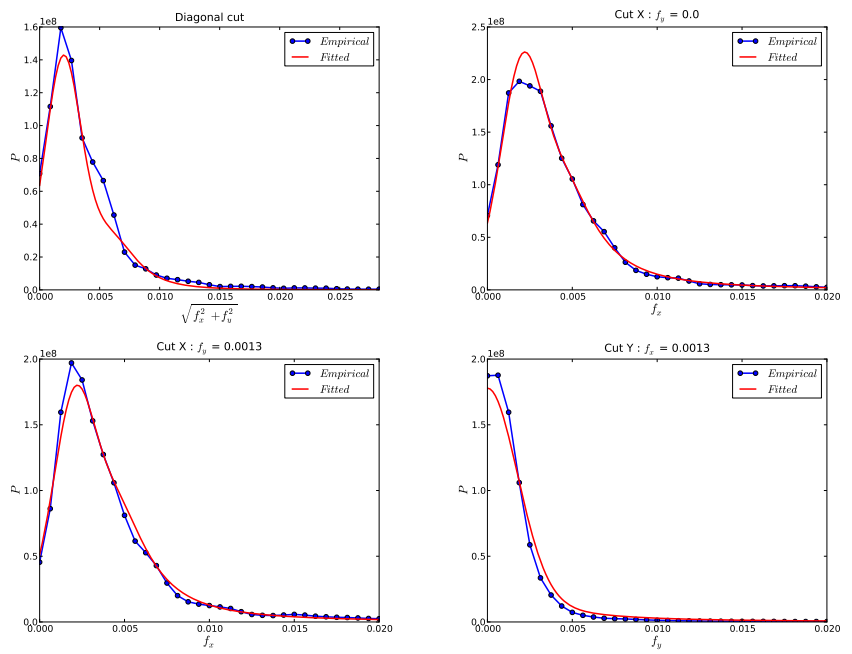

Figure 5: Cut of the periodograms in different directions

but its fluctuation scale is less than the Gaussian component one $\left(0.79 D_{g}-1.23 D_{g}\right.$ vs $\left.2 D_{g}-3 D_{g}\right)$. This result shows that the interaction exists only between 2 or 3 neighbor grains while the fluctuation scale of stress field part on the grain boundaries is in line with the mean size of grains.

Figure 6 shows the evolution of the fitted parameters of the field $\sigma_{I}$ when the number of realization of the stress field increases. Figure 7 shows the evolution of the fitted parameters w.r.t the macroscopic strain level. It appears that the fitted parameters tend to a converged value when at least 25 realizations of the stress field are used for their estimation. In Figure 7, one can observe three deformation steps of the material. In the elastic domain, the correlation lengths are stable when the variance increases. In the domain of elastic-plastic change, the correlation lengths decrease when the variance increases. In the plastic domain, the correlation lengths are stable when the variance increases. The identified stress random field is
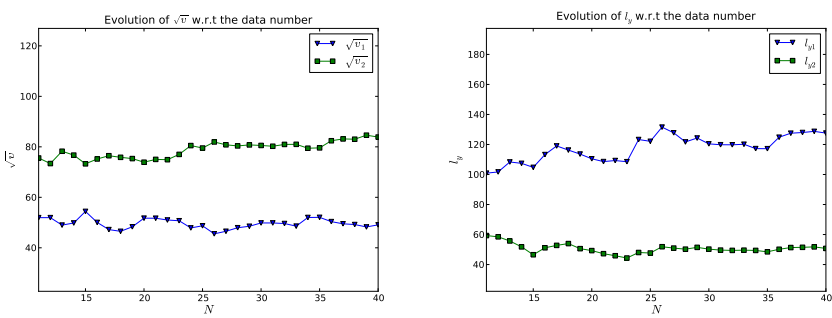

Figure 6: Fitted parameters $\left(\sqrt{v}\right.$ in left and $l_{y}$ in right) of the principal stress field in increase data number

then simulated at each load level using the spectral simulation method $[16,17,18,6]$ to obtain a number of simulated realizations of the field. These realizations are then compared with the resistance stress field presented in the section 4 to estimate the failure probability of the specimen. 

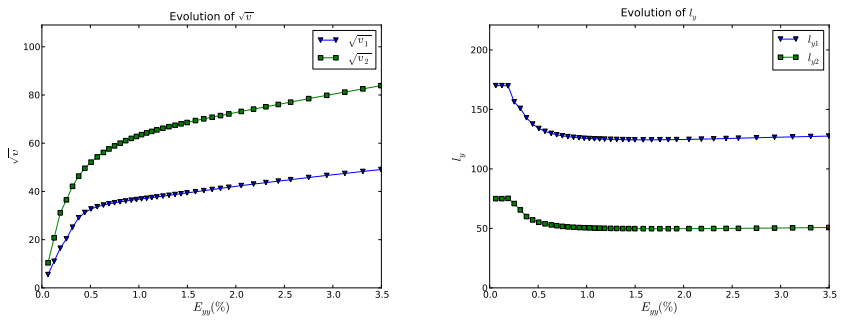

Figure 7: Evolution of fitted parameters $\left(\sqrt{v}\right.$ in left and $l_{y}$ in right) of the principal stress field w.r.t the macroscopic strain level

\section{TAKE INTO ACCOUNT THE STRESS RANDOM FIELD INTO THE LOCAL APPROACH TO FRACTURE}

\subsection{Failure criterion and simulation of carbides distribution}

The brittle fracture of this type of material is assumed to be controlled by the propagation of micro-defects. One assumes that a defect is created by the rupture of the carbides or of the carbides-ferrites joints called the cleavage. The Griffith criterion that defines the minimal stress level that is required to propagate a circular micro-defect of size $r_{c}$ is used as the failure criterion (Eq. 7). The size of the carbides is sampled from a given probability density function according to experimental evidence. In this work, one uses the Ortner model (Eq. (18)) that is proposed in [19].

$F_{R}\left(r_{c}\right)=\mathbb{P}\left(R<r_{c}\right)=\exp \left[-\left(\frac{2 r_{c}}{0,072}\right)^{-2,7}\right]$

Because of the projection and the simulation of the stress field onto a regular post-processing grid, the simulation of carbide distribution must be realized on the same grid through two steps.

- The first step is to sample the number of carbides $N_{c a r}$ on each point of the grid. This is an event sample type with only one available piece of information that is the mean number of carbides calculated by :

$n_{c a r}=\frac{M_{c a r}}{N_{p t s}}=\frac{\mu_{c a r} V}{N_{p t s}}$

where $n_{c a r}$ is the mean number of carbides in each point, $M_{c a r}$ is the total number of carbides in the volume $V$ of the specimen and $N_{p t s}$ is the number of points of the grid and $\mu_{c a r}=$ $7.6 \times 10^{17}$ (carbides $/ \mathrm{m}^{3}$ ) [20] is the volumetric mean number of carbides. Moreover $N_{\text {car }}$ is always positive. As a consequence the number of carbides per grid point $N_{c a r}$ is sampled using the Poisson distribution.

- The second step is to sample the size $r_{c i}(i=$ $\left.1, \ldots, N_{c a r}\right)$ for each carbide in each point. The maximal value of the sampled values of $r_{c i}$ in each point is then used to calculate the resistance stress on this point. Note that there is an upper bound to the carbide size $r_{\max }$ in Ortner's model. Thus we propose to sample $r_{c i}$ by the truncated distribution (Eq. (20)):

$$
F_{R}^{T}\left(r_{c}\right)=\frac{F_{R}\left(r_{c}\right)}{F_{R}\left(r_{\max }\right)}
$$

If the sampled value $r_{c i}>r_{\max }$, it is rejeted and another value will be sampled.

\subsection{Estimation of failure probability}

In Section 4.1, one has simulated the carbides distribution. This allows us to sample a number of realizations of resistance stress field $\sigma_{c}$ on the same projection grid as the one used topost-process the principal stress random field $\sigma_{I}$. In this section, one will use the simulated realizations of these stress fields to estimate the failure probability of the specimen.

One considers that the specimen of size $1000 \times 1000$ corresponds to a $500 \times 500 \times 1 \mu \mathrm{m}^{3}$ specimen of material. The maximal size of carbides is $1 \mu \mathrm{m}$ [20]. Because of low level of loading in the mechanical modeling, and in order to illustrate the method one uses the value of the surface energy about $\gamma_{p}=0.5$ $-2 \mathrm{Jm}^{-2}$. Ones simulates $N=1,000$ realizations for each field $\sigma_{I}$ and $\sigma_{c}$ on the same grid of $256 \times 256$ points.

At each load level $\varepsilon_{i}=0, \ldots, 3.5 \%$, one compares the value of stress fields $\sigma_{I}$ and $\sigma_{c}$ realization by realization and point by point. If in at least one point of the grid $\sigma_{I}>\sigma_{c}$, the specimen is considered to be broken. If among $N=1,000$ samples, $N_{r}^{i}$ ruptures are observed then the failure probability is estimated by a classical Monte Carlo estimate, namely :

$\bar{P}_{R}^{i}=\frac{N_{r}^{i}}{N}$

The $95 \%$ confidence interval of this estimator is defined by [21] :

$P_{R}^{i}=\bar{P}_{R}^{i} \pm 1,96 \sqrt{\frac{\bar{P}_{R}^{i}\left(1-\bar{P}_{R}^{i}\right)}{N}}$

Figure 8 shows the estimated failure probability curve using $N=1,000, r_{\max }=1 \mu \mathrm{m}, \gamma_{p}=1.7 \mathrm{Jm}^{-2}$ and the corresponding confidence interval. This figure shows that the confidence interval is rather small. Thus 1,000 simulations of the stress field appear to be a good compromise in order to obtain the convergence of the estimator.

\section{CONCLUSION}

The main aim of this work is to built a methodology that combines different domains, i.e. polycrystalline aggregates modeling, random field theory and 


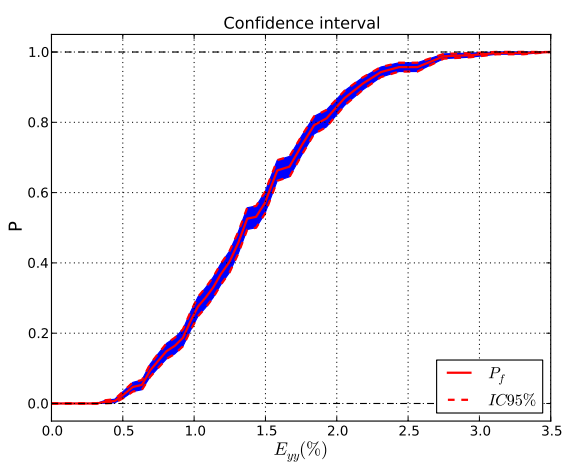

Figure 8: Faillure probability cuve estimated with $N=1000$, $r_{\max }=1 \mu \mathrm{m}, \gamma_{p}=1.7 \mathrm{Jm}^{-2}$

fracture mechanics. This allows us to introduce the spatial variability of the material's microstructure in the local approach to fracture in order to estimate the failure probability of the material. Thus in this work, one limits ourselves in the simple case, i.e. bidimensional polycrystalline aggregates simulation, ergodic stationary Gaussian random field.

However this methodology is open for further developments. In terms of the mechanical modeling, a development to the tridimensional case to account for the triaxiality of loading (such as as in $[7,22]$ ) is neccessary. Secondly the limit at the grain structure scale in the modeling of the polycristalline influences not only the stress field but also the failure probability due to the reduction of the microstructure obstacles. Thus a detailed modeling at the lath packets scale [22] may provide a rather different result. Thirdly the simulation of the grain structure by a purely random Voronoï diagram may not reproduce the material microstructure with full precision. Thus a microstructure construction method from experimental observations $[23,22,24]$ should be investigated.

In terms of identification and simulation of the random field, the ergodic stationary Gaussian hypothesis is clearly very strong. Thus a development towards non-Gaussian (possibly non-stationary) random field representations shall be investigated, see e.g. [25, 26].

\section{REFERENCES}

[1] B. Tanguy. Modélisation de l'essai Charpy par l'approche locale de la rupture - Application au cas de l'acier 16MND5 dans le domaine de transition. PhD thesis, École des Mines ParisTech, 2001.

[2] F.M. Beremin. A local criterion for cleavage fracture of a nuclear pressure vessel steel. Metall. Trans. A, 14A:2277-2287, 1983.

[3] J.P. Mathieu, K. Inal, S. Berveiller, and O. Diard. A micromechanical interpretation of the temperature dependence of Beremin model parameters for French RPV steel. J. Nucl. Mat., 406:97112, 2010.
[4] M. Libert, C. Rey, L. Vincent, and B. Marini. Temperature dependant polycrystal model application to bainitic steel behavior under tri-axial loading in the ductile-brittle transition. Int. J. Solids. Struct., 48(1415):2196 - 2208, 2011.

[5] X.H Dang, B. Sudret, and M. Berveiller. Benchmark of random fields simulation methods and links with identification methods. In M. Faber, J. Köhler, and K. Nishijima, editors, Proc. 11th. Int. Conf. on Applications of Stat. and Prob. in Civil Engineering (ICASP11), Zurich, Switzerland, 2011.

[6] X.H Dang. Identification de la variabilité spatiale des champs de contraintes dans les agrégats polycrystallins et application à l'approche locale de la rupture. $\mathrm{PhD}$ thesis, Université Blais Pascale - Clermont II, 2012.

[7] J.P. Mathieu. Analyse et modélisation micromécanique du comportement et de la rupture fragile de l'acier 16MND5: Prise en compte des hétérogénéités microstructurales. $\mathrm{PhD}$ thesis, École Nationale Supérieure d'Arts et Métiers, 2006.

[8] E. Gilbert. Random subdivisions of space into crystals. Ann. Math. Stat., 33(3):958-972, 1962.

[9] C.B. Barber, D.P. Dobkin, and H. Huhdanpaa. The Quickhull algorithm for convex hulls. $A C M$ Trans. Math. Software, 22(4):469-483, 1996.

[10] P. Franciosi. The concepts of latent hardening and strain hardening in metallic single crystals. Acta Metall., 33(9):1601-1612, 1985.

[11] L. Meric and G. Cailletaud. Single crystal modelling for structural calculation : Part 2 - F.E. implementation. J. Eng. Mat. Tech, 113:171 - 182, 1991.

[12] A.A. Griffith. The phenomena of rupture and flow in solids. Phil. Trans. R. Soc. London, 221:163-198, 1921.

[13] F.J. Harris. On the use of windows for harmonic analysis with the discrete Fourier transform. In Proceedings of the IEEE, volume 66, pages 5183, 1978.

[14] P. Stoica and R. Moses. Spectral analysis of signal. Prentice Hall, 2005.

[15] R.H. Shumway and D.S. Stoffer. Time series analysis and its applications. Springer, 2006.

[16] A. Preumont. Vibrations aléatoires et analyse spectrale. Presses polytechniques et universitaires romandes, 1990. 
[17] M. Shinozuka and G. Deodatis. Simulation of stochastic processes by spectral representation. App. Mech. Rev., 44(4):191-204, 1991.

[18] P. Bocchini and G. Deodatis. Critical review and latest developments of a class of simulation algorithms for strongly non-Gaussian random fields. Prob. Eng. Mech., 23:393-407, 2008.

[19] S.R. Ortner, J. Duff, and D.W. Beardsmore. Characterization of Euro A reference steel for application of $\mathrm{EOH}$ model of brittle fracture. Technical Report SA/EIG/15234/R003, Project PERFECT, SERCO Assurance, 2005.

[20] S.R. Ortner. Factor affecting the shape of the duticle-to-brittle transition. Int. J. of Press. Vessels and Piping, 79:693-700, 2002.

[21] G. Saporta. Probabilités, analyse des données et statistique. Editions Technip, 2nd edition, 2006.

[22] N. Osipov. Génération et calcul de microstructures bainitiques, approche locale intragranulaire de la rupture. $\mathrm{PhD}$ thesis, École des Mines ParisTech, 2007.

[23] D. Jeulin and M. Ostoja-Starzewski. Mechanics of random and multiscale microstructures. Springer, 2001.

[24] B. Kouchmeshky and N. Zabaras. Microstructure model reduction and uncertainty quantification in multiscale deformation processes. Comput. Mater. Sci, 48:213-227, 2010.

[25] C. Soize. Identification of high-dimension polynomial chaos expansions with random coefficients for non Gaussian tensor-valued random fields using partial and limited experimental data. Comput. Methods Appl. Mech. Engrg., 199:2150-2164, 2010.

[26] J. Guilleminot. Modélisation stochastique mésoscopique de milieux aléatoires : application à un polymère renforcé de fibres longues. $\mathrm{PhD}$ thesis, Université de Lille 1, 2008. 